\title{
Entanglement versus Correlations in Spin Systems
}

\author{
F. Verstraete, M. Popp and J.I. Cirac \\ Max-Planck-Institut für Quantenoptik, 85748 Garching, Germany
}

(Dated: October 25, 2018)

\begin{abstract}
We consider pure quantum states of $N \gg 1$ spins or qubits and study the average entanglement that can be localized between two separated spins by performing local measurements on the other individual spins. We show that all classical correlation functions provide lower bounds to this localizable entanglement, which follows from the observation that classical correlations can always be increased by doing appropriate local measurements on the other qubits. We analyze the localizable entanglement in familiar spin systems and illustrate the results on the hand of the Ising spin model, in which we observe characteristic features for a quantum phase transition such as a diverging entanglement length.
\end{abstract}

PACS numbers: 03.67.Mn, 03.67.-a, 73.43.Nq, 05.50.+q

The mathematical description of multiparticle quantum systems plays an important role in several branches of physics. The main difficulty stems from the fact that the number of parameters needed to describe a quantum state grows exponentially with the number of particles. However, sometimes it is possible to capture the most relevant physical properties by describing these systems in terms of very few parameters. This is the case, for example, in quantum statistics, where two-particle correlations play a fundamental role. They allow us to understand several complex physical phenomena, like phase transitions. Furthermore, they give rise to concepts like correlation length, which quantifies a very intuitive property of these systems.

Multiparticle systems are also of central interest in the field of quantum information and, in particular, the quantification of the entanglement contained in quantum states. The reason is that entanglement is the physical resource to perform some of the most important quantum information tasks, like quantum information transfer (cfr. teleportation) or quantum computation.

Given the common interest of quantum statistical mechanics and quantum information in multiparticle systems it is natural to try to describe the physical phenomena, like quantum phase transitions, appearing in (e.g.) spin systems from the point of view of entanglement. The main restriction one encounters is the fact that there exist very few measures of multiparticle entanglement with a clear physical meaning. In any case, since entanglement measures correlations (for pure states), one would expect that reasonable entanglement measures be intimately connected to the correlation functions widely used in the context of quantum statistical mechanics. This is not the case, however, if one studies the behavior of the entanglement of formation between two separate spins after tracing out the rest [1, 2, 3, 4, 5]. Although this approach exhibits a very pronounced (universal) behavior of this quantity at the transition point, it rapidly vanishes as the distance between the spins goes beyond 3 or 4 , and thus it is not related to the correlations possessed by the state. Note also that the approach by Vidal et al. [6], in which they study the scaling of entanglement of a block of spins with the other ones, does not quantify the entanglement as a resource (that could be used, for example, for teleportation).

In this Letter we introduce a new concept which we call Localizable Entanglement (LE). On the one hand, this quantity has a very well defined physical meaning which treats entanglement as a truly physical resource. On the other, it establishes a very close connection between entanglement and correlation functions, as one would naturally expect. The LE of two particles is the maximal amount of entanglement that can be localized in these two particles, on average, by doing local measurements in the rest of the particles. The LE naturally leads to the definition of entanglement length, which measures the typical length scale at which the LE decays. The LE has an operational meaning which applies to situations in which out of some multiparticle entangled state one would like to concentrate as much entanglement as possible in two particular particles. This occurs, for example, in the context of quantum repeaters [7] or in the context of quantum transport with spin systems (spintronics [8]). The determination of the LE is a formidable task since it involves an optimization over all possible local measurement strategies, and thus cannot be determined in general. We have nevertheless managed to determine tight upper and lower bounds. The first ones stem from considering joint measurements on the rest of the spins, which is closely related to the concept of entanglement of assistance [9]. The second and more interesting ones can be derived by proving that there always exist local measurement which do not decrease the existing correlations between the two spins, something that despite its generality, up to our knowledge has not been considered in the context of quantum information.

The usefulness of these findings will be illustrated on the hand of the entanglement present in the ground states of standard spin Hamiltonians. Let us however first con- 
sider a simple example involving the $N$-qubit GHZ-state:

$$
|G H Z\rangle=\frac{1}{\sqrt{N}}(|00 \cdots 0\rangle+|11 \cdots 1\rangle) .
$$

In this case, the LE is maximal as it is possible to create a Bell-state between two arbitrary qubits by measuring the other qubits in the $|+\rangle,|-\rangle$ basis (here $| \pm\rangle=(|0\rangle \pm$ $|1\rangle) / \sqrt{2})$. The existence of these quantum correlations could also have been revealed by studying the classical correlation functions

$$
Q_{\alpha \beta}^{i j}=\left\langle\psi\left|\sigma_{\alpha}^{i} \otimes \sigma_{\beta}^{j}\right| \psi\right\rangle-\left\langle\psi\left|\sigma_{\alpha}^{i}\right| \psi\right\rangle\left\langle\psi\left|\sigma_{\beta}^{j}\right| \psi\right\rangle,
$$

where $i, j$ denote the positions of the spins under interest and $\alpha, \beta$ label the Pauli matrices. The correlation in the $\alpha=\beta=z$-direction is the maximal possible one. It will indeed be shown that correlation functions yield lower bounds to the LE. However, in general the presence or absence of classical correlations only gives a coarse-grained picture of the entanglement that ought to be created between two distant spins. It is easy to find examples of highly entangled quantum states exhibiting no classical correlations whatsoever between any pair of spins. As an example, consider the so-called cluster states [10], obtained by the unitary evolution of an initially separable state under the action of the Ising Hamiltonian:

$$
|\psi\rangle=\frac{1}{2^{N / 2}}\left(\otimes_{i=1}^{N-1}\left(|0\rangle_{i} \sigma_{z}^{(i+1)}+|1\rangle_{i}\right)\right)\left(|0\rangle_{N}+|1\rangle_{N}\right)
$$

When $N \geq 5$, all reduced 2-qubit density operators are proportional to the identity and hence no correlations exist between any two spins. However, suitable local measurements on any $N-2$ qubits can always create a Bell-state between the two remaining ones [10], hence indicating maximal LE.

Let us next give a formal definition of the LE. Consider a pure state $|\psi\rangle$ of $N$ spins. Then the localizable entanglement $E_{i j}(\psi)$ is variationally defined as the maximal amount of entanglement that can be created (i.e. localized), on average, between the spins $i$ and $j$ by performing local measurements on the other spins. More specifically, every measurement basis specifies a pure state ensemble $\mathcal{E}=\left\{p_{s},\left|\phi_{s}\right\rangle\right\}$ consisting of at least $2^{(N-2)}$ elements counted by the index $s$. In this notation $p_{s}$ denotes the probability to obtain the two-spin state $\left|\phi_{s}\right\rangle$ after performing the measurement $|s\rangle$ on the assisting spins of our $\mathrm{N}$ partite spin system. The LE is then defined as

$$
E_{i j}=\max _{\mathcal{E}} \sum_{s} p_{s} E\left(\left|\phi_{s}\right\rangle\right),
$$

where $E\left(\left|\phi_{s}\right\rangle\right)$ denotes the entanglement of $\left|\phi_{s}\right\rangle$. As we deal with pure states of two qubits, all entanglement measures are essentially equivalent, and in order to make the connection with correlation functions, we will measure the entanglement on the hand of the concurrence [1]: indeed, it can readily be checked that the maximal correlation function [18] for a pure state of two qubits coincides with the concurrence, given by $C(|\psi\rangle=a|00\rangle+b|01\rangle+c|10\rangle+d|11\rangle)=2|a d-b c|$. Note that the behavior of the LE as defined in terms of the entropy of entanglement would be very similar 19 .

Due to the variational definition, the LE is very difficult to calculate in general. Moreover, in typically large spin systems one does not have an explicit parametrization of the state under interest, but just information about the classical 1- and 2-particle correlation functions (which parameterize completely the 2-qubit reduced density operator). It would therefore be interesting to derive tight upper and lower bounds to the LE solely based on this information. The upper bound can readily be obtained using the concept of entanglement of assistance [9], which would correspond to the LE if global or joint measurements were allowed on the other spins. It was shown in 14] that the entanglement of assistance can be calculated as follows: given the reduced density operator $\rho_{i j}$ and a square root $X, \rho_{i j}=X X^{\dagger}$, then the entanglement of assistance $E_{i j}(\psi)$ as measured by the concurrence is equal to the trace norm $\operatorname{Tr}\left|X^{T}\left(\sigma_{y} \otimes \sigma_{y}\right) X\right|$.

The lower bound is more subtle, and will be shown to follow from the following interesting theorem: given a (pure or mixed) state of $N$ qubits with classical correlation $Q_{\alpha \beta}^{i j}$ between the spins $i$ and $j$ and directions $\alpha, \beta$, then there always exist directions in which one can measure the other spins such that this correlation do not decrease, on average. This automatically implies that there always exist local measurements that increase (or keep) the classical correlations. Surprisingly, this very general theorem seems not to have been noticed before, and is interesting on its own. It could be very useful in the context of cryptography (e.g. multipartite distribution of common randomness [15]).

Let us next proof this theorem. Note that it is sufficient to consider mixed states of three qubits. Let us parameterize a mixed 3-qubit density operator by four $4 \times 4$ blocks

$$
\rho=\left[\begin{array}{cc}
\rho_{1} & \sigma \\
\sigma^{\dagger} & \rho_{2}
\end{array}\right] .
$$

Without loss of generality, let us consider the $Q_{z z}^{12}$ correlations. The original correlations are completely determined by the diagonal elements of the density operator $\rho_{1}+\rho_{2}$. A von Neumann measurement in the $| \pm\rangle:=\cos (\theta / 2)|0\rangle \pm \sin (\theta / 2) \exp ( \pm i \phi)|1\rangle$ basis on the third qubit results in the hermitian unnormalized 2-qubit operators

$$
\begin{aligned}
X_{ \pm}= & \frac{\rho_{1}+\rho_{2}}{2} \pm \cos (\theta) \frac{\rho_{1}-\rho_{2}}{2} \\
& \pm \sin (\theta)\left(\cos (\phi) \frac{\sigma+\sigma^{\dagger}}{2}+\sin (\phi) \frac{i\left(\sigma-\sigma^{\dagger}\right)}{2}\right) .
\end{aligned}
$$

Defining $p_{ \pm}=\operatorname{Tr}\left(X_{ \pm}\right)$, we have to prove that there always exist parameters $\theta, \phi$ such that the correlation, on 
average, does not decrease:

$p_{+}\left|Q_{z z}\left(X_{+} / p_{+}\right)\right|+p_{-}\left|Q_{z z}\left(X_{-} / p_{-}\right)\right| \geq\left|Q_{z z}\left(X_{+}+X_{-}\right)\right|$.

Without loss of generality we can assume that $\alpha=$ $Q_{z z}\left(X_{+}+X_{-}\right)$is positive. Removing the absolute value signs and parameterizing

$$
\bar{x}:=[\cos (\theta) ; \sin (\theta) \cos (\phi) ; \sin (\theta) \sin (\phi)],
$$

some straightforward algebra yields the sufficient inequality

$$
\bar{x}^{T}\left[\alpha\left(\bar{c}-\frac{\bar{\beta}}{\alpha}\right)\left(\bar{c}-\frac{\bar{\beta}}{\alpha}\right)^{T}+Q-\frac{\bar{\beta} \bar{\beta}^{T}}{\alpha}\right] x \geq 0
$$

where $\bar{c}$ is such that $p_{ \pm}=\left(1 \pm \bar{c}^{T} \bar{x}\right) / 2$, and $\bar{\beta}, Q$ are defined as $3 \times 1$ and $3 \times 3$ blocks of the matrix

$$
S=R^{T}\left(\sigma_{y} \otimes \sigma_{y}\right) R=\left[\begin{array}{cc}
\alpha & \bar{\beta}^{T} \\
\bar{\beta} & Q
\end{array}\right] .
$$

Here $R$ is the real $4 \times 4$ matrix whose columns consist of the diagonal elements of the matrices $\left(\rho_{1}+\rho_{2}\right),\left(\rho_{1}-\rho_{2}\right)$, $\left(\sigma+\sigma^{\dagger}\right), i\left(\sigma-\sigma^{\dagger}\right)$.

Due to Sylvester's law of inertia [12], we know that $S$ has two positive and two negative eigenvalues. Now $Q-\bar{\beta} \bar{\beta}^{T} / \alpha$ is the Schur complement of $\alpha$, and hence corresponds to a principal $3 \times 3$ block of the matrix $S^{-1}$. Due to the interlacing properties of eigenvalues of principal blocks [12], it follows that it has either two positive and one negative eigenvalue or two negative and one positive one. And this of course ensures that there always exists an $\bar{x}$ such that the inequality (10) is fulfilled, completing the proof.

Note that the proof is constructive and allows to determine a measurement strategy that would at least achieve the bound reported. Note also that exactly the same proof applies when correlations between larger blocks of spins would be considered [13].

Let us now show how this theorem yields a lower bound to the LE. Given an initial pure state of $N$ qubits, we know that the measurement of the first, second, ... N3 'th qubit can be chosen such that on average the final correlations do not decrease. But we end up with a pure state of two qubits, for which the concurrence is equal to the maximal correlation. A lower bound to the LE is therefore given by the maximal correlation function, and following the previous theorem, there is always a constructive way of determining a measurement strategy that achieves this lower bound. Surprisingly, we will see that this lower bound seems to be the exact value for the LE in the case of many systems of interest.

The previous findings can readily be applied to the study of entanglement in translational invariant ground and excited states of spins arranged in a regular lattice. In quantum statistics and more specifically in the study of quantum phase transitions, the correlation length is the canonical parameter of interest. The concept of LE readily lends itself to define the related entanglement length $\xi_{E}$ as the typical length scale at which it is possible to create Bell states by doing local measurements on the other spins. More specifically, the entanglement length is finite if and only if the the LE $E_{i, i+n} \simeq \exp \left(-n / \xi_{E}\right)$ decays exponentially in $n$, and the entanglement length $\xi_{E}$ is defined as the constant in the exponent in the limit of an infinite system (see also Aharonov [16]):

$$
\xi_{E}^{-1}=\lim _{n \rightarrow \infty}\left(\frac{-\log E_{i, i+n}}{n}\right)
$$

Note that a diverging correlation length automatically implies a diverging entanglement length and hence longrange quantum correlations, although the converse is not necessarily true 20$]$.

More specifically, we have studied ground states of two spin interaction Hamiltonians with parity symmetry $\otimes_{i=1}^{N} \sigma_{z}^{i}$ of the form:

$$
\mathcal{H}=-\sum_{i, j} \sum_{\alpha=x, y, z} \gamma_{\alpha}^{i j} \sigma_{\alpha}^{i} \sigma_{\alpha}^{j}-\sum_{i} \gamma^{i} \sigma_{z}
$$

with coupling coefficients $\gamma_{x}^{i j} \geq \gamma_{y}^{i j} \geq 0$ (note that this model also includes spin systems in higher dimensional lattices). Extensive numerical calculations on systems of up to 20 qubits showed that our lower bound is always very close to the LE, and typically is even equal to the exact value of the LE [13]: this is surprising and highlights the power of the given lower bound. Note also that whenever the parity symmetry is present, the upper and lower bounds can easily be calculated in terms of the correlation functions:

$$
\begin{aligned}
\max \left(Q_{x x}^{i j}, Q_{y y}^{i j}, Q_{z z}^{i j}\right) & \leq E_{i j} \leq \frac{\sqrt{s_{+}^{i j}}+\sqrt{s_{-}^{i j}}}{2} \\
s_{ \pm}^{i j} & =\left(1 \pm\left\langle\sigma_{z}^{i} \sigma_{z}^{j}\right\rangle\right)^{2}-\left(\left\langle\sigma_{z}^{i}\right\rangle \pm\left\langle\sigma_{z}^{j}\right\rangle\right)^{2}
\end{aligned}
$$

As an illustration, we consider the ground state of the Ising Hamiltonian $\left(\gamma_{\alpha}^{i j}=\lambda \delta_{\alpha, x} \delta_{j, i+1} ; \gamma^{i}=1\right)$, which has been solved exactly [17] and exhibits a quantum phase transition at $\lambda=1$. In this case, the maximal classical correlation is always given by $Q_{x x}$, and we conjecture that it is equal to the LE (for more details, we refer to [13]). For $\lambda<1$, the LE is small as the ground state is almost separable, and the entanglement length is finite (see Fig. 1) as the LE decreases exponentially with the spin distance. At the quantum critical point $\lambda=1$, the behavior of the LE changes drastically, as it decreases polynomially $E_{i, i+n} \sim n^{-1 / 4}$, thus leading to a diverging entanglement length $\xi_{E}$. In the case $\lambda>1$ we also get $\xi_{E}=\infty$, as the LE saturates to a finite value given by $M_{x}^{2}=1 / 4\left(1-\lambda^{-2}\right)^{1 / 4}$. Indeed, the ground state is then close to the GHZ-state. This behavior is illustrated in Fig. 1. 

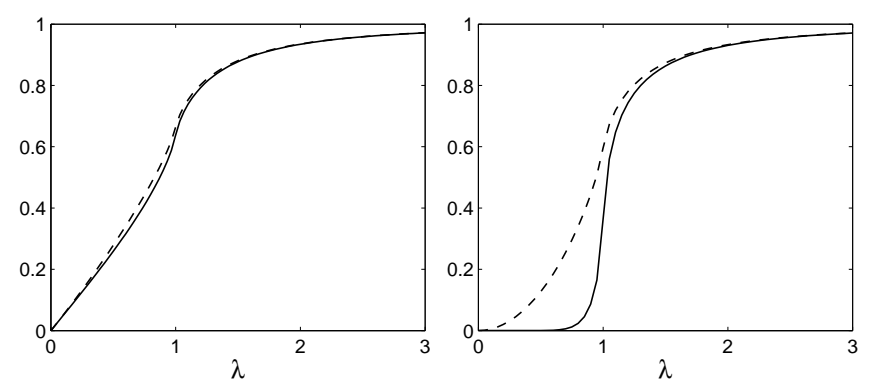

FIG. 1: Localizable Entanglement $E_{i j}$ and Correlation function $Q_{x x}^{i j}$ (solid) and upper bound (dashed) as a function of the coupling parameter $\lambda$ for the infinite Ising chain with spin distance $n=1$ (left) and $n=10$ (right).

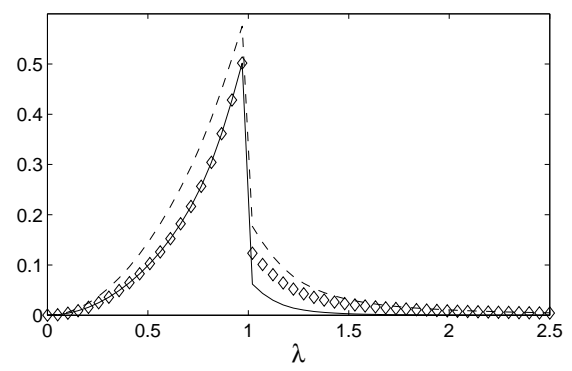

FIG. 2: Correlation function $Q_{x x}^{i, i+4}$ (solid), Localizable Entanglement $E_{i, i+4}$ (diamonds), and its upper bound (dashed) as a function of the coupling parameter $\lambda$ for the ground state of the finite Ising chain $(N=14)$ in the case of broken parity symmetry by a small perturbating magnetic field in the $x$-direction.

In a more realistic setup however, the parity symmetry of the Ising Hamiltonian will be broken by a perturbation and the ground state for large coupling will also be separable. Indeed, the energy gap between the lowest energy states with different parity decays exponentially in the number of qubits in the region $\lambda>1$, and henceforth the ground state becomes a superposition of these two states. The calculation of its LE and the corresponding lower and upper bounds are depicted in Fig. 2.

These results give a clear illustration of the intimate connection between classical correlations and entanglement in the case of ground states of translational invariant Hamiltonians. The plethora of results concerning classical correlation functions, such as diverging correlation length at quantum phase transitions, can now be interpreted from the perspective of quantum information theory; one could argue that the status of classical correlations has been lifted to the one of (useful) quantum correlations.

In conclusion, we have introduced the notion of localizable entanglement. It has a nice operational meaning as it quantifies the amount of useful entanglement that can be created between two spins by doing local measurements on all other spins. We proved that classical correlation functions always provide lower bounds to the LE, showing that the presence of classical correlations is sufficient to be able to create Bell-like quantum correlations. As a side-product, we proved that classical correlations in multipartite mixed quantum states can always increase by doing appropriate measurements on the other qubits. Finally, we demonstrated the usefulness of these concepts in the context of spin systems on a lattice, provided a natural definition of entanglement length, and showed that it diverges at a quantum phase transition. Further generalizations and applications will be presented elsewhere [13].

We acknowledge interesting discussions with J. Garcia Ripoll. This work was supported in part by the E.C. (projects RESQ and QUPRODIS) and the Kompetenznetzwerk "Quanteninformationsverarbeitung" der Bayerischen Staatsregierung.

[1] A. Osterloh et al., Nature 416, 608 (2002).

[2] T.J. Osborne and M.A. Nielsen, Phys. Rev. A 66, 032110 (2002)

[3] K.M. O'Connor and W.K. Wootters, Phys. Rev. A 63, $052302(2001)$

[4] P. Zanardi and X. Wang, J. Phys. A 35, 7947 (2002)

[5] M.C. Arnesen, S. Bose and V. Vedral, Phys. Rev. Lett. 87, 017901 (2001).

[6] G. Vidal et al., Phys.Rev.Lett. 90, 227902 (2003).

[7] H.J. Briegel et al, Phys. Rev. Lett. 81, 5932 (1998).

[8] D.D. Awschalom, D. Loss, and N. Samarth, Semiconductor Spintronics and Quantum Computation, SpringerVerlag, Berlin, 2002.

[9] D. P. DiVincenzo et al. quant-ph/9803033

[10] H.J. Briegel and R. Raussendorf, Phys. Rev. Lett. 86: 910 (2001).

[11] W.K. Wootters, Phys. Rev. Lett. 80:2245 (1998).

[12] R.A. Horn and C.R. Johnson, Matrix Analysis, Cambridge University Press, 1985.

[13] M. Popp, F. Verstraete and J.I. Cirac, in preparation.

[14] T. Laustsen, F. Verstraete, and S. J. van Enk, Quant. Inf. and Comp. 3, 64 (2003).

[15] I. Devetak and A. Winter, quant-ph/0304196

[16] D. Aharonov, Phys. Rev. A 62, 062311 (2000).

[17] P. Pfeuty, Ann. Phys. 57, 79 (1970).

[18] The basis with maximal possible correlation can in general be found by calculating the singular value decomposition of the $3 \times 3$ matrix $Q_{\alpha \beta}$, and the largest singular value corresponds to the maximal possible correlation.

[19] For a given concurrence $C$, the entropy of entanglement is given by $f(C)=H\left(\left(1+\sqrt{1-C^{2}}\right) / 2\right)$ with $H(x)$ the Shannon entropy. Due to the convexity of $f(C)$, the following bounds hold: $f\left(\sum_{s} p_{s} C_{s}\right) \leq \sum_{s} p_{s} f\left(C_{s}\right) \leq$ $\sum_{s} p_{s} C_{s}$.

[20] This leaves open the possibility of Hamiltonians exhibiting a phase transition as indicated by a diverging entanglement length at the critical point but with a correlation length remaining finite. 